\title{
Bibliothèques scientifiques
}

\section{Christian Jacob}

\section{(2) OpenEdition \\ Journals}

\section{Édition électronique}

URL : https://journals.openedition.org/rbnu/1596

DOI : 10.4000/rbnu. 1596

ISSN : 2679-6104

\section{Éditeur}

Bibliothèque nationale et universitaire de Strasbourg

\section{Édition imprimée}

Date de publication : 1 novembre 2014

Pagination : 8-9

ISBN : 9782859230548

ISSN : 2109-2761

\section{Référence électronique}

Christian Jacob, «Bibliothèques scientifiques », La Revue de la BNU [En ligne], 10 | 2014, mis en ligne le 01 novembre 2014, consulté le 17 mai 2021. URL : http://journals.openedition.org/rbnu/1596 ; DOI : https://doi.org/10.4000/rbnu.1596

\section{(c) $10(0)$}

La Revue de la BNU est mise à disposition selon les termes de la Licence Creative Commons Attribution - Pas d'Utilisation Commerciale - Partage dans les Mêmes Conditions 4.0 International. 


\section{Bibliothèques scientifiques}

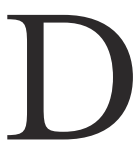

ans un article qui a fait date ${ }^{1}$, Germaine Aujac, éminente spécialiste de la science grecque, s'interroge sur le fait qu'un certain nombre de traités de physique, mécanique, mathématique et astronomie parvenus jusqu'à nous depuis l'Antiquité grecque, sous une forme plus ou moins complète, ont été écrits à peu près à la même époque, au $3^{\mathrm{e}}$ siècle avant notre ère, par des auteurs appartenant à une même génération, sous le règne des Ptolémée : Autolycos de Pitane, Euclide, Aristarque de Samos, Archimède, Apollonios de Pergé. On pourrait aussi ajouter deux auteurs de la seconde moitié du $2^{\mathrm{e}}$ siècle avant notre ère, Hypsiclès d'Alexandrie et Théodose de Bithynie. Germaine Aujac avance une hypothèse pour expliquer ce phénomène : la survivance de ces traités, même sous forme fragmentaire, serait due à une politique délibérée du bibliothécaire d'Alexandrie, Eratosthène de Cyrène, lui-même polymathe, astronome, cartographe et géographe. En d'autres termes, Eratosthène aurait pris soin de réunir dans la biblothèque des copies des travaux des meilleurs spécialistes de son temps, avec lesquels, pour certains d'entre eux du moins, il était en relation épistolaire. Il aurait également entrepris un travail de correction éditoriale de ces manuscrits, sur le modèle de l'édition des textes littéraires menée à la bibliothèque.

Sans doute ne faudrait-il pas imaginer à Alexandrie une section des livres scientifiques sur le modèle de nos établissements contemporains, mais il est significatif qu'Eratosthène, à côté de la vocation prioritaire de la bibliothèque à accueillir le patrimoine littéraire de l'hellénisme et l'ensemble des travaux philologiques et antiquaires qui prenaient appui sur lui, ait veillé à réunir des exemplaires des travaux scientifiques les plus récents, contribuant à perpétuer le programme intellectuel des écoles philosophiques athéniennes, en particulier l'Académie platonicienne qu'il fréquenta pendant plusieurs années et le Lycée aristotélicien.

Ce moment alexandrin pourrait ainsi introduire notre réflexion sur les bibliothèques scientifiques, qu'il faut distinguer des bibliothèques de scientifiques. Ces dernières, si elles renferment des ouvrages spécialisés dans un ou plusieurs champs techniques, peuvent aussi accueillir des livres relevant d'autres disciplines, des sciences humaines et d'érudition, de la littérature, de la théologie ou de la philosophie. Comme toutes bibliothèques personnelles, elles reflètent à la fois la singularité d'une trajectoire de vie, d'une curiosité et d'un itinéraire intellectuel, et les déterminations collectives d'un milieu familial, social, professionnel, confessionnel, situé au carrefour de multiples normes et influences. L'inventaire d'une bibliothèque personnelle, on le sait, livre des informations stratifiées et complexes, non seulement sur les ouvrages effectivement acquis, lus et utilisés, mais aussi sur la sphère d'intérêts, sur la représentation de soi qu'elle pouvait déployer, sur les réseaux de sociabilité intellectuelle tissés par les dons et contredons de livres ou de tirés à part.

Les bibliothèques scientifiques sont d'un autre ordre. Ce sont les ensembles de textes et de documents constitués et mobilisés dans la pratique d'une discipline particulière, dans son enseignement comme dans son enrichissement par la recherche. Mais au-delà des collections de telle institution ou de tel laboratoire particulier, c'est le rapport plus général des sciences au livre et à l'écriture qui est en jeu. Quel est le statut du livre, de la revue, des collections de livres et de revues dans les différentes disciplines scientifiques et leurs ramifications ? Quels types d'espaces intellectuels, quelles profondeurs temporelles déploient ces collections ? Et enfin, quelles sont les formes du travail sur les textes scientifiques, quelles sont les pratiques de lecture, et en quoi la référence aux écrits joue-t-elle un rôle opératoire dans le processus de recherche et de découverte ?

Il n'y a évidemment pas de réponse globale, ni même disciplinaire. Les questions générales que nous formulons ici ne prennent sens que dans des lieux et des moments particuliers, dans des projets intellectuels singuliers, mais aussi dans des contextes professionnels, politiques et économiques situés. Suivre au plus près les usages et les courbes de vie des écrits scientifiques est évidemment une nécessité stratégique, tant pour les bibliothèques qui tentent aujourd'hui de maîtriser les coûts des abonnements, tant imprimés qu'en " bouquets numériques ", que pour les chercheurs qui lisent, écrivent et publient. Choisir les formes de communica- 
tion scientifique les mieux adaptées et les plus efficaces est crucial tant pour la diffusion des résultats que pour l'affirmation de leur antériorité et de la propriété intellectuelle qui s'y rattache, ainsi que celle de leurs enjeux commerciaux et industriels. Hiérarchiser les revues selon un certain nombre d'indicateurs contribue aussi à l'établissement de différents régimes d'autorité qui rejaillissent sur les contenus publiés comme sur les chercheurs "publiants ". Enfin, l'accélération exponentielle des communications numériques, l'accroissement des données et l'" overflow " informationnel rendent plus que jamais nécessaire l'existence d'instruments de maîtrise et de contrôle de ces flux vertigineux.

Sur ces différents fronts, les bibliothèques de chercheurs, de laboratoires, d'universités et d'instituts, mais aussi les bibliothèques nationales et de lecture publique sont confrontées aux mêmes défis : à quoi servent les livres et les revues scientifiques ? Est-ce que les scientifiques lisent plus ou moins que les praticiens des sciences humaines et sociales, est-ce qu'ils lisent différemment ? Comment articuler la spécialisation la plus extrême et les ouvrages généralistes, les fonds destinés aux étudiants, doctorants, " post-docs » et spécialistes les plus pointus ? Comment s'imbriquent les pratiques expérimentales, les démarches théoriques, l'écriture, l'enseignement ?

Aux oppositions tranchées, aux clivages du sens commun, il conviendrait de substituer une observation fine et située des pratiques, des usages, des représentations, des dispositifs de publication, de circulation et d'enseignement. Au bloc monolithique de telle ou telle science considérée dans son ensemble, il faut préférer les jeux d'échelle et de découpage qui permettent de construire les objets, hypothèse, expérience, déduction, induction, résultat avéré, ainsi que les régimes d'autorité qui les valident (revues internationales avec comités de lecture, etc.). À côté de l'évidence qu'un article est toujours un article, il faut tenir compte des différences considérables introduites par les modes de diffusion, bouquet d'abonnements, open access, archive ouverte, modèle freemium, modèle gold, mais aussi par les pressions particulières exercées sur les chercheurs par les instances d'évaluation.

Je reviendrai pour conclure à mon point de vue d'Alexandrin, qui me permettra de poser deux ultimes questions.

La première est celle de la profondeur historique des bibliothèques scientifiques. Est-il utile de conserver les revues ou les ouvrages les plus anciens, même s'ils sont dépassés ? Comment se négocie la transition entre sciences et histoire des sciences ? Il n'y a pas de réponse unique ici non plus. On pourrait imaginer que les mathématiques contemporaines sont l'une des disciplines les plus évolutives, avec une date de péremption des publications très rapprochée, où les écrits, les modèles, les raisonnements deviennent vite obsolètes en étant remplacés par les travaux les plus récents. Or il n'en est rien, et les mathématiciens peuvent trouver un intérêt à se reporter à la généalogie des tentatives de résolution d'un problème ancien et à reprendre des voies et des raisonnements dont on n'a pas exploité tout le potentiel heuristique. Les observations des voyageurs du passé, de même, peuvent rester d'un immense intérêt pour les sciences naturelles d'aujourd'hui, ainsi que les descriptions de phénomènes célestes et météorologiques, ou encore les cartes anciennes pour instruire les politiques contemporaines d'aménagement du territoire et de prévention des risques naturels.

La seconde réflexion, liée, porte sur la manière dont certains savoirs se prêtent à la production de livres-bibliothèques ou plutôt de dispositifs qui, d'une certaine façon, réemploient les savoirs antérieurs dans un livre qui rend la consultation de la bibliothèque inutile. Ces ouvrages peuvent prendre deux formes antithétiques. La première est la réduction extrême, à travers la réécriture, le résumé, la formalisation, le passage du singulier au général, du spécimen à la classe, de l'événement au phénomène, l'occultation des processus heuristiques pour s'en tenir aux résultats, la mise à jour des savoirs aux dépens de leur généalogie : on reconnaît là les dispositifs du manuel, de la carte, des tableaux, des arbres de classification, des formules mathématiques, physiques ou chimiques. La seconde forme est celle de l'expansion et de la mise en ordre encyclopédique, en rassemblant la plus grande quantité de savoir possible, d'informations, de données, d'observations, prélevées dans les livres antérieurs : ce sont là les dynamiques de la compilation, de la vulgarisation, d'ouvrages qui peuvent répondre à la raréfaction des livres antérieurs pour en préserver, en absorber et en transmettre l'essentiel. Ce sont là deux formes de bibliothèques scientifiques, l'une privilégiant la vision synoptique, la visibilité et l'intelligibilité, l'autre déroulant des fils analogiques et digressifs selon des principes d'ordre qui peuvent être alphabétique, chronologique, géographique, linguistique, typologique, et invitant à des navigations sans fin dans les océans du savoir, réunis dans un même ouvrage, conjoignant l'expansion et le quadrillage.

$\mathrm{Au}$ fond, deux formes différentes de bibliothèques portables, d'arches de Noé graphiques pour les savoirs du monde...

\section{Christian Jacob}

\section{Notes}

1 - Germaine Aujac, Eratosthène, premier éditeur de textes scientifiques?, in Pallas, XXIV (Annales de l'Université de Toulouse Le Mirail, N. S., tome XIII, 1977, fasc. 3), p. 3-24 